Check for updates

Cite this: J. Mater. Chem. A, 2020, 8 , 14243

Received 5th March 2020

Accepted 24th June 2020

DOI: 10.1039/d0ta02590a

rsc.li/materials-a

\section{D printing-directed auxetic Kevlar aerogel architectures with multiple functionalization options $\uparrow$}

\author{
Qingqing Cheng, ${ }^{\text {ab }}$ Yang Liu, (D) ${ }^{c}$ Jing Lyu, ${ }^{\mathrm{b}}$ Qiang Lu, (D) ${ }^{\mathrm{d}}$ Xuetong Zhang (D) *be \\ and Wenhui Song (iD *e
}

Auxetic architectures with a negative Poisson's ratio have attracted increasing attention due to their intriguing physical properties and numerous promising applications and recent advancements in manufacturing techniques. However, fabrication of three-dimensional (3D) polymeric auxetic architectures with a tailored hierarchically porous structure and desired physical/mechanical properties remains challenging. Herein, 3D nanofibrous Kevlar aerogel architectures with porosity at multiple scales have been designed and fabricated through a new additive manufacturing strategy, i.e., integration of direct ink writing and freeze-casting with non-toxic solvent-based inks followed by special drying techniques. The highly porous 3D nanofibrous Kevlar aerogel architectures achieve excellent mechanical properties with an ultralow density (down to $11.9 \mathrm{mg} \mathrm{cm}^{-3}$ ) and large specific surface area (up to $350 \mathrm{~m}^{2}$ $\mathrm{g}^{-1}$ ). The Poisson's ratio is tunable in a wide range, spanning from -0.8 to 0.4 , by adjusting the spatial arrangement of the designed pattern struts. Finally, these nanofibrous Kevlar aerogel architectures have been further functionalized into hydrophobic, luminescent and thermoresponsive architectures by using fluorocarbon resin, functional dyes and organic phase-change materials respectively. The multifunctional auxetic aerogel architectures demonstrate potential for a broad range of applications.

\section{Introduction}

Aerogels are synthesized from gels by replacement of the liquid component with a gas. ${ }^{1}$ They appear as porous solid-state nanomaterials with extremely low density, high specific surface area, low thermal conductivity and excellent dielectric properties, having great importance in various applications including thermal management, energy storage, catalysis, environmental treatment, electromagnetic wave shielding. ${ }^{2-5}$

${ }^{a}$ School of Nano-Tech and Nano-Bionics, University of Science and Technology of China, Hefei 230026, P. R. China

${ }^{b}$ Suzhou Institute of Nano-Tech and Nano-Bionics, Chinese Academy of Sciences, Suzhou 215123, P. R. China. E-mail: xtzhang2013@sinano.ac.cn

'Department of Materials, Imperial College London, SW7 2AZ, UK

${ }^{d}$ National Engineering Laboratory for Modern Silk, Soochow University, Suzhou 215123, P. R. China

${ }^{e}$ Centre for Biomaterials in Surgical Reconstruction and Regeneration, Division of Surgery and Interventional Science, University College London, London NW3 2PF, UK. E-mail:w.song@ucl.ac.uk

$\uparrow$ Electronic supplementary information (ESI) available: Characterization of inks, photos, DSC curves, rheological properties, XRD spectra, FTIR spectra, schematic diagram, XFEM modeling, simulated stress-strain curve, tensile energy absorption distribution, simulated plot of the energy per volume as a function of strain, tensile stress-strain curve, nitrogen adsorption-desorption isotherms and pore volume, TGA curves, movies of the cryo-3D printing process, tensile mechanical response, droplet transport, and shape memory of the 3D printed Kevlar aerogel architecture (AVI). See DOI: 10.1039/d0ta02590a
Recently, many tremendous improvements have been accomplished. For example, a layer of a silica aerogel has been demonstrated experimentally and theoretically to make Mars habitable for photosynthetic life ${ }^{6}$ Aerogels have even been used on the Stardust spacecraft to capture cosmic dust. ${ }^{7}$ All these aerogels were synthesized from the sol-gel process together with subsequent supercritical fluid drying. However, such traditional technologies exhibited the following shortcomings: (1) lack reproducible customization over the 3D structures of aerogels, and (2) lack the ability to easily fabricate complex aerogel structures. By contrast, 3D cryo-printing followed by supercritical fluid drying can overcome the above-mentioned shortcomings and can produce next-generation aerogels with tailored structures in light of the merits of the accurate, rapid, programmable and scalable characteristics of 3D printing (i.e. additive manufacturing).

On the other hand, auxetic architectures with a negative Poisson's ratio describe the degree of transverse deformation of a given material when it is axially stretched or compressed..$^{\mathbf{8 1 0}}$ This unusual mechanical behaviour has been shown to occur in carbon allotropes, ${ }^{11}$ foams,${ }^{12}$ microporous polymers, ${ }^{13,14}$ laminates ${ }^{\mathbf{1 5 , 1 6}}$ and other crystalline materials with hinged crystal structures. ${ }^{17}$ Such designable auxetic architectures possess intriguing mechanical properties including superior shear resistance, indentation resistance, fracture toughness and damping performance, ${ }^{18-20}$ promising a diverse range of 
applications, particularly under stringent conditions such as in aerospace, defence and smart systems as well as biomedical devices. The auxetic behaviour of architectures can respectively take place at the macro, micro, nano or even molecular level depending on material architectures designed and specific manufacturing techniques applied. Several strategies have been extensively explored to demonstrate different capacities of design and fabrication of auxetic architectures at different scales including mould-assistance, ${ }^{21}$ direct cutting or milling, ${ }^{22}$ soft lithography, ${ }^{23}$ nanomembrane technology, ${ }^{24}$ template growth, ${ }^{13}$ sol-gel process, ${ }^{25}$ and freezing-defrosting process ${ }^{26}$ as well as additive manufacturing. ${ }^{18,20}$ Nevertheless, designing and manufacturing auxetic architectures with mutli-scale structures is still challenging.

By considering the advantages of aerogels in the design and processing of auxetic architectures, it is envisaged that auxetic architectures will be endowed with a more hierarchically porous structure and a maximised range of physical properties. Despite various challenges of integration of the aerogel synthetic process with manufacture of the complex structure of auxetic architectures, new auxetic architectures made from carbon, inorganic or metallic aerogels through unconventional process techniques have been reported recently. ${ }^{27-29}$ For example, a hyperbolically patterned 3D graphene aerogel, with a negative Poisson's ratio, was obtained by the oriented growth of ice crystals during freeze casting. ${ }^{27}$ Using such $3 \mathrm{D}$ graphene aerogels as templates, hyperbolically architectured boron nitride ceramic aerogels were also synthesized having a negative Poisson's ratio and a negative linear thermal expansion coefficient. ${ }^{28}$ Lin and co-workers ${ }^{29}$ reported silver nanowire aerogels with tunable Poisson's ratio by integrating drop-on-demand inkjet printing and freeze casting. Nevertheless, to date, there have been few reports on the fabrication of polymeric aerogels with auxetic architectures in the literature. Therefore, the development of appropriate methodology for fabricating polymeric auxetic aerogels, with a designed structure and multi-functionality in a cost-effective manner for practical applications, is desired and value-added.

Herein, a new feasible and versatile 3D printing method has been developed by integrating direct ink writing (DIW) and freeze-casting with dimethyl sulfoxide (DMSO)-based inks. Different from previous water-based freeze-casting or direct micro extrusion, ${ }^{30,31}$ this approach exhibits an advanced capacity of manufacturing auxetic architectures from a diverse range of polymer aerogels or nanomaterial aerogels with a higher resolution, faster printing rate and lower energy cost. 3D polymeric aerogel based auxetic architectures made from a Kevlar nanofibre DMSO suspension, as an exemplar, demonstrate superior mechanical properties with tunable in-plane Poisson's ratios ranging from negative $(-0.8)$ to positive $(0.4)$ values by varying unit-cell structures with inherent ultralow densities and high specific surface areas. Furthermore, a 3D Kevlar aerogel auxetic architecture (3D-KAAA) was further functionalized by taking advantage of its unique auxetic structures and properties. Multifunctional 3D-KAAAs and composites with superhydrophobicity, photochromicity and thermoresponsive shape memory were demonstrated. This work opens an avenue for the design and fabrication multi- functional auxetic architectures and auxetic-composites for potential diverse applications in many emerging fields.

\section{Experimental}

\section{Materials}

DMSO and ethanol were purchased from Sinopharm Chemical Reagent Co., and Kevlar 1000D was purchased from Dongguan SOVETL Co. Ltd. Potassium hydroxide $(\mathrm{KOH})$ and polyethylene glycol (PEG-2K) were purchased from Aladdin Co.; paraffin with ceresin (wax) was purchased from Huayong Paraffin Co. Ltd., Shanghai, China. Fluorocarbon (FC) resin was obtained from Sino-Fluorine Tech. Ltd, Shenzhen, China. Graphene oxide (GO) and silver nanowires (AgNWs) were synthesized by our group, ${ }^{32,33}$ and multi-walled carbon nanotubes (MWCNTs) were purchased from Macklin Co. Deionized water $\left(18.2 \mathrm{M} \Omega \mathrm{cm}^{-1}\right.$ ) was obtained from a Millipore Milli-Q system. All the chemicals were used without further purification.

\section{Preparation of DMSO-based inks}

Aramid nanofibre (ANF)/DMSO dispersions with a concentration of $0.5,1$, and $2 \mathrm{wt} \%$ were prepared using a method described elsewhere by our group. ${ }^{34,35}$ Typically, 2 wt $\%$ ANF/ DMSO dispersions were prepared by dispersing $2 \mathrm{~g}$ of bulk Kevlar in $98 \mathrm{~g}$ of DMSO in the presence of $2 \mathrm{~g}$ of $\mathrm{KOH}(\mathrm{KOH}$ is almost insoluble), and then magnetically stirred for 1 week at least at room temperature to form a uniform dark-red dispersion of ANFs. AgNWs, MWCNTs and GO were directly dispersed in DMSO at a concentration of $10 \mathrm{mg} \mathrm{ml}^{-1}$, respectively.

\section{Characterization of inks}

The morphology of ANFs was characterized by scanning electron microscopy (SEM, S-4800, Hitachi, Japan), atomic force microscopy (AFM, Dimension Icon, Bruker, Germany) and transmission electron microscopy (TEM, FEI, Hillsboro, USA). The molecular weight $\left(M_{\mathrm{W}}\right)$ and polydispersity index (PDI) were determined by gel permeation chromatography (GPC, 1515, Waters, USA). The phase transition of the ink was characterized at a heating and cooling rate of $10{ }^{\circ} \mathrm{C} \mathrm{min}^{-1}$ by using a differential scanning calorimeter (DSC2500, TA Instruments, USA). The isothermal crystallization kinetics of the ink was also tested by quenching the ink to crystallization temperatures of $0,-2$, and $-10{ }^{\circ} \mathrm{C}$ respectively at a cooling rate of $200{ }^{\circ} \mathrm{C} \mathrm{min}^{-1}$. The steady shearing and dynamic rheological properties of the inks were characterized by using a rotational rheometer (RS6000, Haake, Germany) with a gap of $1 \mathrm{~mm}$. Two tests were carried on the ANF/DMSO inks at $25{ }^{\circ} \mathrm{C}:{ }^{30}$ (1) steady shear rate sweep to measure the apparent viscosity at a shear rate ranging from 0.1 to $100 \mathrm{~s}^{-1}$, and (2) dynamic stress sweep to measure the storage modulus $\left(G^{\prime}\right)$ and loss modulus $\left(G^{\prime \prime}\right)$ at a shear stress amplitude ranging from 0.1 to $100 \mathrm{~Pa}$ with a constant frequency of $1 \mathrm{~Hz}$.

\section{D printing process}

The printing process was performed using a custom-made robotic deposition system with a 3-axis desktop controlled by three-step motors using G-code commands. A cold plate was 
connected to a thermostatic water bath (DC-4506, FDL Tech, Nanjing, China) and an automatic dispensing system (MY3131T, Meiyuan Tech, Shenzhen, China) with a suck-back dispensing valve mounted on the $x-y-z$ motor. One end of the dispensing valve had a microneedle, with an inner diameter of $330 \mu \mathrm{m}$ (Nordson EFD, USA), and the other end was connected to an oil free air compressor (OTS-550, OTS, Taizhou, China). To obtain the ANF/DMSO cryogels, the prepared $2 \mathrm{wt} \%$ ANF/DMSO dispersion was loaded into the dispensing valve though a constant extruding pressure of 1-2 bar, and then printed onto the cold plate at $-10{ }^{\circ} \mathrm{C}$ according to the unit-cell structure designed by computer aided design (CAD). The initial nozzle height from the substrate was optimised at around $250 \mu \mathrm{m}$ to ensure sufficient adhesion onto the substrate, and the moving speed of the nozzle was $14 \mathrm{~mm} \mathrm{~s}^{-1}$. The as-printed 3D ANF cryogels were then solvent-exchanged with cold water of $4-6{ }^{\circ} \mathrm{C}$ and washed with ethanol to remove water from the pores. Subsequently, supercritical drying with $\mathrm{CO}_{2}$ was carried out to obtain the 3D Kevlar aerogel architectures.

\section{Modification of 3D Kevlar aerogel architectures}

For the preparation of hydrophobic 3D Kevlar aerogel architectures, the original architecture was immersed in FC solution directly followed by ambient pressure drying. ${ }^{32}$ The preparation of the photochromic aerogel architecture was achieved by soaking the 3D Kevlar aerogel architecture in different alcoholic solutions including metal complex dyes or rhodamine B for $24 \mathrm{~h}$. For the preparation of phase change architectures, wax or PEG-2K was completely melted at $80{ }^{\circ} \mathrm{C}$, and then the 3D Kevlar aerogel architecture was immersed and maintained at $80{ }^{\circ} \mathrm{C}$ for another $6 \mathrm{~h}$. Subsequently, the excess PCM adhered on the surface was removed through transferring the samples to filter paper. The impregnation process was repeated 3 times.

\section{Determination of Poisson's ratios}

Both ends of the fabricated samples were fastened between two pieces of a polystyrene sheet using bolts and nuts, and then loaded into an Instron 3365 tensile testing machine. The top end was attached to a movable single-axis positioning system and the bottom end attached between two fixed steel blocks. Each sample was tested in a vertical setup and an axial load was applied, with a tensile rate of $1 \mathrm{~mm} \mathrm{~min}^{-1}$. The in-plane movement of these $3 \mathrm{D}$ architectures in the longitudinal and transverse direction was monitored by using a high-resolution digital camera. Each photo from the strain test was fed to GetData Graph Digitizer software to accurately measure the displacement in the transverse and longitudinal direction, respectively. ${ }^{19}$ The definition of Poisson's ratio used for validation is the negative ratio of the transverse strain, $\varepsilon_{x}$, to the longitudinal strain, $\varepsilon_{y}$, along the loading direction: ${ }^{20}$

$$
\nu=-\varepsilon_{y} / \varepsilon_{x}
$$

The applied deformation was measured by the engineering strain, i.e., $\varepsilon=\Delta L / L_{0}$, where $\Delta L$ is the change in the distance between two points initially defined by the distance $L_{0}$. The experimentally measured points are the center of the sample edge. Three experiments were conducted for each sample group.

\section{Characterization and measurements}

The morphology of the samples was characterized by using an scanning electron microscope (SEM, S-4800, Hitachi, Japan) with an acceleration voltage of $5 \mathrm{kV}$. Before SEM examination, the samples were coated with $\mathrm{Au}$ by using a sputter-coater at a current of $20 \mathrm{~mA}$ for $2 \mathrm{~min}$. The pore size distribution and average pore diameter were analyzed by the Barrett-JoynerHalenda (BJH) nitrogen adsorption and desorption method (ASAP 2020, Micromeritics, USA) and the specific surface area and pore volume were determined by the Brunauer-EmmettTeller (BET) method, based on the amount of $\mathrm{N}_{2}$ adsorbed at pressure $0.05<P / P_{0}<0.3$. The structure of the samples was recorded by X-ray diffraction (XRD, D8 advance, Bruker AXS, Germany) with $\mathrm{Cu} \mathrm{K} \alpha$ generated at $40 \mathrm{kV}$ and $40 \mathrm{~mA}$ and Fourier transform infrared spectroscopy (FTIR 5700, FL, USA) over 64 scans recoded with a resolution wavelength of $4 \mathrm{~cm}^{-1}$. Thermal stability was determined by using a thermal gravimetric analyzer (TGA, 209F1, NETZSCH, Germany) with a heating rate of 10 $\mathrm{K} \mathrm{min}^{-1}$ in a nitrogen atmosphere. The stress-strain curves were recorded by using an Instron 3365 tensile testing machine. The water contact angle measurements were performed at room temperature by using a contact angle meter (OCA 15EC Data Physics Instruments GmbH, Germany). The infrared photos were taken with a MinIR (M1100150, SAT, China) camera.

\section{Extended finite element method (XFEM) modeling of the 3D- KAAA at the macroscopic scale}

XFEM modeling of the macroscopic structure of the 3D-KAAA was conducted using the ABAQUS platform. The length scales in the $x-$ $y-z$ three directions of the reconstructed auxetic-structure were set to the same aspect ratio as the experimental specimen. Due to the disallowance of the quadratic element for finite element analysis (FEA) subroutine, the calculation was implemented based on the C3D8 (cube element with full 8 integration points) linear brick elements to model the crack propagation inside the internal strut. The whole auxetic-structure was defined as the XFEM crack growth region with no specified contact properties and crack location involved. 288744 linear elements with fine meshes of the maximum size of $5 \mathrm{~mm}$ were used so that the accurate crack propagation was captured for the auxetic-structure. A single Kevlar aerogel fibre with an elastic modulus of $10.4 \pm 2.2 \mathrm{MPa}$ and Poisson's ratio of 0.1-0.3 was used for each point of the 3D-KAAA in the simulation. ${ }^{35}$ Maximum principal stress was considered as 3.30 MPa obtained at the initial point of fracture. The displacement at failure was set as $0 \mathrm{~mm}$, which meant that the stress dropped directly to $0 \mathrm{MPa}$ once the fracture occurred. The viscosity coefficient of 0.001 was set as the relaxed parameter for damage stabilization and further stress equilibrium of the model.

XFEM modeling of the section of a single strut of the 3D-KAAA at the microscopic scale

The section of a single strut of the auxetic-structure at the microscopic scale was modeled using entangled beam arrays. 
Under the local tensile strain, the section experienced the inner pressure from the edge. 232016 C3D10 quadratic tetrahedral elements with a maximum mesh ratio of 0.002 were applied to the section to ensure the mesh continuity along each Kevlar fibre and the model accuracy. A Young's modulus of 90-131 GPa and Poisson's ratio of 0.36 of the Kevlar nanofibres in the strut were applied based on ref. 36 and properties of commercial Kevlar $49 .{ }^{37}$ In addition, a maximum principal strain threshold was applied in the simulation to ensure that the single strut would not exceed the ultimate strength level of $3.67 \mathrm{GPa}$.

\section{Results and discussion}

The additive manufacturing process for a freestanding 3D-KAAA is described in Scheme 1, and it involves the formulation of aramid nanofibre dispersion ink, 3D printing the 3D-KAAA, supercritical drying and a post modification process. ANF was made from polyparaphenylene terephthalamide (PPTA), well known by its trade name, Kevlar, with exceptional mechanical performance, chemical and solvent resistance, thermooxidative stability, flame retardancy and a negative coefficient of thermal expansion. $^{36,38}$ The ANF/DMSO dispersion was made by dispersing Kevlar microfibres in $\mathrm{DMSO} / \mathrm{KOH}$ solution, from which aerogel films ${ }^{34}$ and fibres ${ }^{35}$ were already developed inhouse. The chemistry and the $M_{\mathrm{W}} / \mathrm{PDI}$ of the Kevlar fibre and ANFs as well as the morphology of ANFs are demonstrated in Fig. S1-S4 in the ESI. $\uparrow$ The ANF/DMSO dispersion is under the balance of the $\pi-\pi$ stacking, electrostatic repulsion, van der Waals force as well as physical entanglement among the polymer chains, meaning that the ANF/DMSO dispersion is homogeneous and stable for a long time. ${ }^{36}$ The optimisation of printable ANF/DMSO inks was carried out using a custom-made 3D micro-extruder with a cooling system.

A new universal DIW 3D printing system with a cryo-stage was designed and developed. DIW is a robust and versatile additive manufacturing technique based on continuous microextrusion of a suspension or ink with a specific range of viscoelasticity at low cost. It has been widely used to prototype and manufacture various types of materials which are not commercially available or in small quantities and difficult to

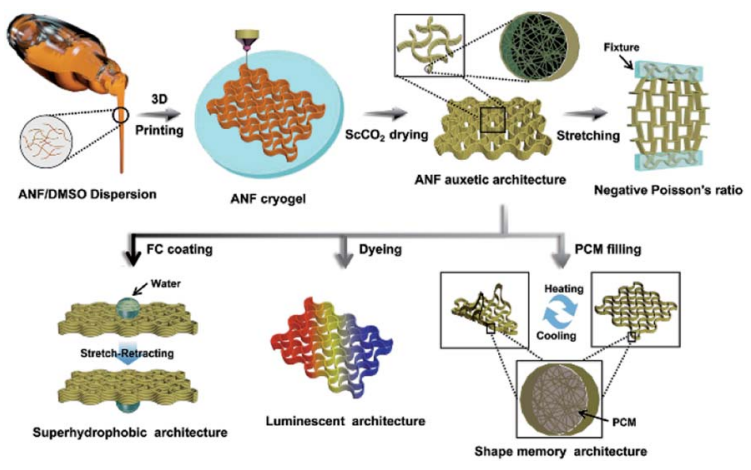

Scheme 1 Schematic illustration of the 3D printing process of a Kevlar aerogel auxetic architecture with a negative Poisson's ratio, series of post-treatments and further functionalization. print using other printing techniques. Some multifunctional 3D engineered materials with a controlled morphology and superior properties have been manufactured by DIW. ${ }^{30,31}$ Waterbased inks are commonly used in DIW in combination with freeze-casting processes; however, several limitations still remain, including the low freezing point $\left(0^{\circ} \mathrm{C}\right)$, high crystallization energy $\left(0.43 \mathrm{~kJ} \mathrm{~g}^{-1}\right)$ and relatively low boiling point (100 ${ }^{\circ} \mathrm{C}$ ) of water plus limited material selection. In contrast, DMSO has distinct physicochemical properties including a high freezing point $\left(18.54{ }^{\circ} \mathrm{C}\right)$, low crystallization energy $\left(0.19 \mathrm{~kJ} \mathrm{~g}^{-1}\right)$, and high boiling point $\left(189^{\circ} \mathrm{C}\right)$, which means that DMSO-based inks would be more stable, and easier to freeze at relatively high temperature with lower energy cost, more favourable for the DIW process (Fig. 1a). Another advantage is that DMSO, a polar aprotic solvent and a strong hydrogen bond acceptor, not only dissolves a wide range of organic materials, but also shows good dispersibility with a number of nanomaterials including MWCNTs, GO, AgNWs, and ANFs, indicating a universal attribute for 3D printing (Fig. S5 in the ESI† and Fig. 1b). On the other hand, DMSO is a non-toxic solvent, widely used as a cryoprotectant for cell preservation to prevent ice formation during the freezing process and in medical treatment for oral or transdermal drug delivery. ${ }^{39}$ Consequently, in comparison with popular cellulose nanofibre aqueous inks, ${ }^{\mathbf{4 0}} \mathrm{ANF} / \mathrm{DMSO}$ inks need neither high pressure homogenization nor introduction of highly charged chemical groups to enhance the uniformity and dispersibility.

The crystallization behaviour of the solvent plays an important role in this $3 \mathrm{D}$ printing process. The rapid cooling at the cryo-stage $\left(-10{ }^{\circ} \mathrm{C}\right)$ during printing would induce the supercooling phenomenon of the printed ink, with evidence of the crystallization temperature $\left(T_{\mathrm{c}}\right)$ of $\mathrm{DMSO},-0.08{ }^{\circ} \mathrm{C}$, and the ANF/DMSO dispersion, $4.25^{\circ} \mathrm{C}$, both of which are much higher than the $T_{\mathrm{c}}$ of water $\left(-22.5^{\circ} \mathrm{C}\right)$ tested by differential scanning calorimetry (DSC) (Fig. 1c and S6 in ESI $\dagger$ ). The upshift of crystallisation temperature of the ink compared to that of pure DMSO may be attributed to the nucleation effects of ANFs in DMSO. The isothermal crystallization behaviour of $2 \mathrm{wt} \% \mathrm{ANF} /$ DMSO dispersion at different temperatures $\left(0,-2\right.$, and $\left.-10{ }^{\circ} \mathrm{C}\right)$ was also investigated by DSC, indicating that the crystallization half-time was increased with an increase of isothermal crystallization temperature. The crystallization profiles at $-2{ }^{\circ} \mathrm{C}$ and $-10{ }^{\circ} \mathrm{C}$ almost overlapped with similar crystallization halftimes, around 58 seconds (Fig. 1d, S7, and S8 in the ESI and Movie S1 $\dagger$ ). Another primary challenge in the approach was the preparation of inks with appropriate viscoelasticity in order to sustain the weight and hold the shape of a printed part without deformation while exhibiting good adhesion to the previous layers. ${ }^{30,31,41}$ As shown in Fig. S9 in the ESI, $\dagger$ without addition of any rheological modifiers, pronounced shear thinning behaviours were observed for the ANF/DMSO dispersions at different concentrations $(0.5-2 \mathrm{wt} \%)$, in that their viscosity decreased by several orders of magnitude with the increasing shear rate from 0.1 to $100 \mathrm{~s}^{-1}$, indicating efficient flow of the dispersion when it is extruded through a fine deposition nozzle. The highest viscosity of $c a$. 22.3 $\mathrm{Pa} \mathrm{s}$ at a shear rate of $1 \mathrm{~s}^{-1}$ was observed from the $2 \mathrm{wt} \% \mathrm{ANF} / \mathrm{DMSO}$ dispersion. Stress sweep 


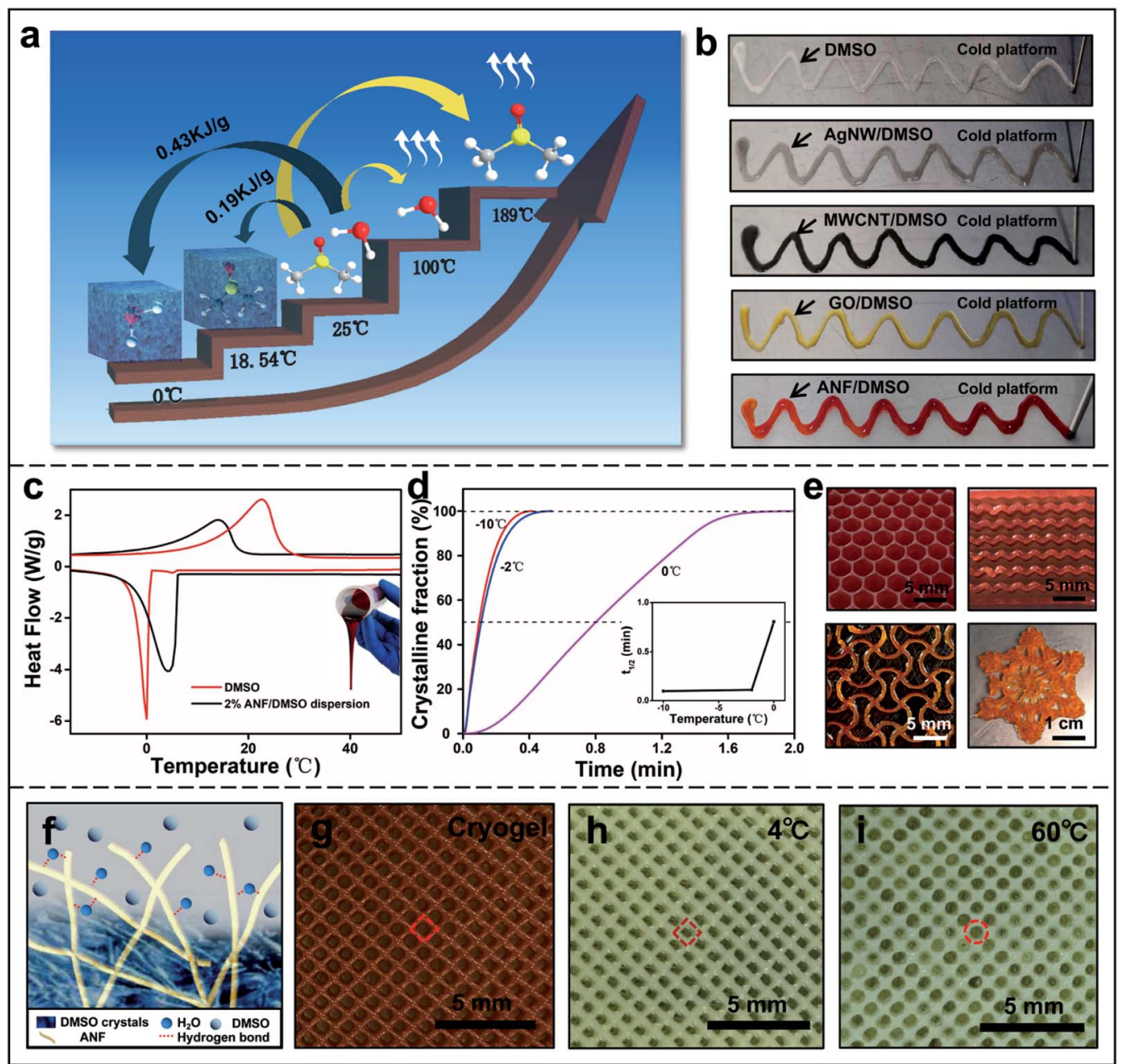

Fig. 1 (a) Comparison of the phase transition temperature and crystallisation enthalpy of DMSO and water. (b) Wave-shaped patterns depicted by DMSO-based inks with various materials on a cold plate. (c) DSC curves of DMSO and the $2 \mathrm{wt} \% \mathrm{ANF} / \mathrm{DMSO}$ dispersion (scan rate $10^{\circ} \mathrm{C}$ min ${ }^{-1}$ ). The inset in (c) is a digital photo of the $2 \mathrm{wt} \%$ ANF/DMSO dispersion. (d) Isothermal crystallization curves of the $2 \mathrm{wt} \%$ ANF/DMSO dispersion at different temperatures $\left(0,-2,-10^{\circ} \mathrm{C}\right)$. The inset in (d) is the plot of the crystallization half-time as a function of isothermal crystallization temperature. (e) Digital photos of printed ANF cryogels with different patterns. (f) Schematic diagram of the formation of the ANF hydrogel from the ANF cryogel through solvent exchange at the solid (DMSO)-liquid (water) interface. ( $g$-i) Digital photos of a printed woodpile structure consisting of layer-by-layer orthogonal grids of the ANF cryogel (g) and hydrogel (h and i) after solvent exchange at the temperature $4{ }^{\circ} \mathrm{C}$ ( $\mathrm{h}$ ) and $60{ }^{\circ} \mathrm{C}$ (i) respectively.

measurements revealed that the dispersions with different concentrations exhibited elasticity-dominated flow behaviour $\left(G^{\prime}>G^{\prime \prime}\right)$ at low shear stress. Specifically, the $2 \mathrm{wt} \%$ ANF/DMSO dispersion exhibited a plateau value of the storage modulus $G^{\prime}$ $\sim 8000 \mathrm{~Pa}$ and yield stress $\tau_{y} \sim 70 \mathrm{~Pa}$, respectively, ${ }^{30}$ exhibiting a rapid recovery of an elastic-gel-like response required during $3 \mathrm{D}$ printing. Therefore, the $2 \mathrm{wt} \%$ ANF/DMSO dispersion was chosen because its viscosity, flow behaviour and high storage modulus met the essential requirements for printable ink, which must be extrudable through a narrow nozzle while still being able to retain structural integrity after printing.

By taking advantage of optimised ANF/DMSO ink and robust DIW combined with freeze-casting, freestanding 3D Kevlar cryogel constructs with a variety of architectures, such as woodpile, honeycomb and snowflake patterns, were printed, demonstrating the capacity of fabricating complex structures with high resolution and excellent reproducibility (Fig. 1e, S10, and S11 in the ESI and Movie S2†). Upon subsequent solvent exchange between the ANF/DMSO cryogel with water, more additional hydrogen bonds between water and $\mathrm{NH}$ functional groups on ANFs were formed due to the energetically favourable $\mathrm{N}-\mathrm{H} \cdots \mathrm{O}=\mathrm{C}$ interaction in water. ${ }^{36,38}$ Ultimately, the $3 \mathrm{D}$ Kevlar hydrogel architectures consisting of entangled nanofibres were formed (Fig. S12, ESI $\dagger$ ). It is noteworthy that during the solvent exchange process, mixing water with DMSO could generate mixing heat which might thaw the frozen printed structure before the formation of ANF hydrogels. Therefore, the temperature of the exchanged water bath had a significant role in maintaining the printing resolution. To optimise the water temperature, the water was controlled at different temperatures $\left(4{ }^{\circ} \mathrm{C}\right.$ and $\left.60^{\circ} \mathrm{C}\right)$ during exchange with DMSO from a freshly printed woodpile cryogel. The result demonstrated that cryogels 
exchanged with water at low temperature $\left(4{ }^{\circ} \mathrm{C}\right)$ had retained their orthogonal shape and resolution of the printed grids better than that at $60{ }^{\circ} \mathrm{C}$ at which the melting and fusion between the printed struts were observed. It is clear that the slow and localised solvent exchange at the liquid-solid interface between water and DMSO crystals at low temperatures could slow down or prevent the potential fast liquid-liquid diffusion at elevated temperatures, at which the ANF/DMSO cryogel started to melt (Fig. 1f-i and S13 in the ESI $\dagger$ ).

In order to prevent the 3D printed Kevlar woodpile structure from shrinking under capillary force during removal of the solvent, either a supercritical $\mathrm{CO}_{2}$ drying or freeze drying process was applied. The aerogel woodpile structure possessed an ultralow density, only $26.8 \mathrm{mg} \mathrm{cm}^{-3}$ and $17.4 \mathrm{mg} \mathrm{cm}^{-3}$ after supercritical drying and freeze drying, respectively, yet showed excellent mechanical properties (Fig. S14-S16, ESI $\dagger$ ). The compressive stress and strain profile of the Kevlar aerogel woodpile is shown in Fig. 2a; three deformation regimes including an initial Hookean elastic region at a strain of $\varepsilon<$ $13 \%$, a plateau after the yield point and final densification regime. The compressive Young's modulus, $470 \mathrm{kPa}$, was measured from the slope of the elastic regime, nearly 25 times higher than that of a polyacrylonitrile (PAN) nanofibre- assembled cellular aerogel (20 kPa with a density of $9.6 \mathrm{mg}$ $\left.\mathrm{cm}^{-3}\right)^{\mathbf{4 2}}$ and 5 times higher than that of pure silk fibroin aerogel monoliths (90 kPa with a density of $\left.20 \mathrm{mg} \mathrm{cm}{ }^{-3}\right) .{ }^{43}$ The compressive yield stress of $70 \mathrm{kPa}$ is more than 15 times higher than that of $3 \mathrm{D}$ graphene bulk with cellular networks $(4.5 \mathrm{kPa}$ with a density of $\left.6.1 \mathrm{mg} \mathrm{cm}^{-3}\right)^{44}$ and 350 times higher than that of a $3 \mathrm{D}$ printed silver nanowire aerogel $(0.2 \mathrm{kPa}$ with a density of $\left.12.6 \mathrm{mg} \mathrm{cm}^{-3}\right) .{ }^{29}$ The elastic region corresponds to the bending of the ANF nanofibres, the relatively flat plateau is attributed to the postbuckling deformation of the nanofibres, and the rapid stress increase region is due to the densification of the various pores in 3D printed aerogels. ${ }^{45,46}$ Therefore, the Kevlar aerogel woodpile structure with a density of $26.8 \mathrm{mg} \mathrm{cm}^{-3}$ could withstand a compressive load of $9.8 \mathrm{~N}$ (compressive stress of 28.9 $\mathrm{kPa}$ ) at the critical compression strength (Fig. 2b). The superior elasticity of the woodpile strip was also demonstrated through its reversible helical twist, subsequent folding and rolling (Fig. 2c).

The scanning electron micrographs in Fig. $2 \mathrm{~d}-\mathrm{f}$ reveal a unique multiscale network structure, contributing to the mechanical properties of such lightweight aerogel materials. The printed layers were superimposed precisely on top of one another following the design of the 3D orthogonal pattern as

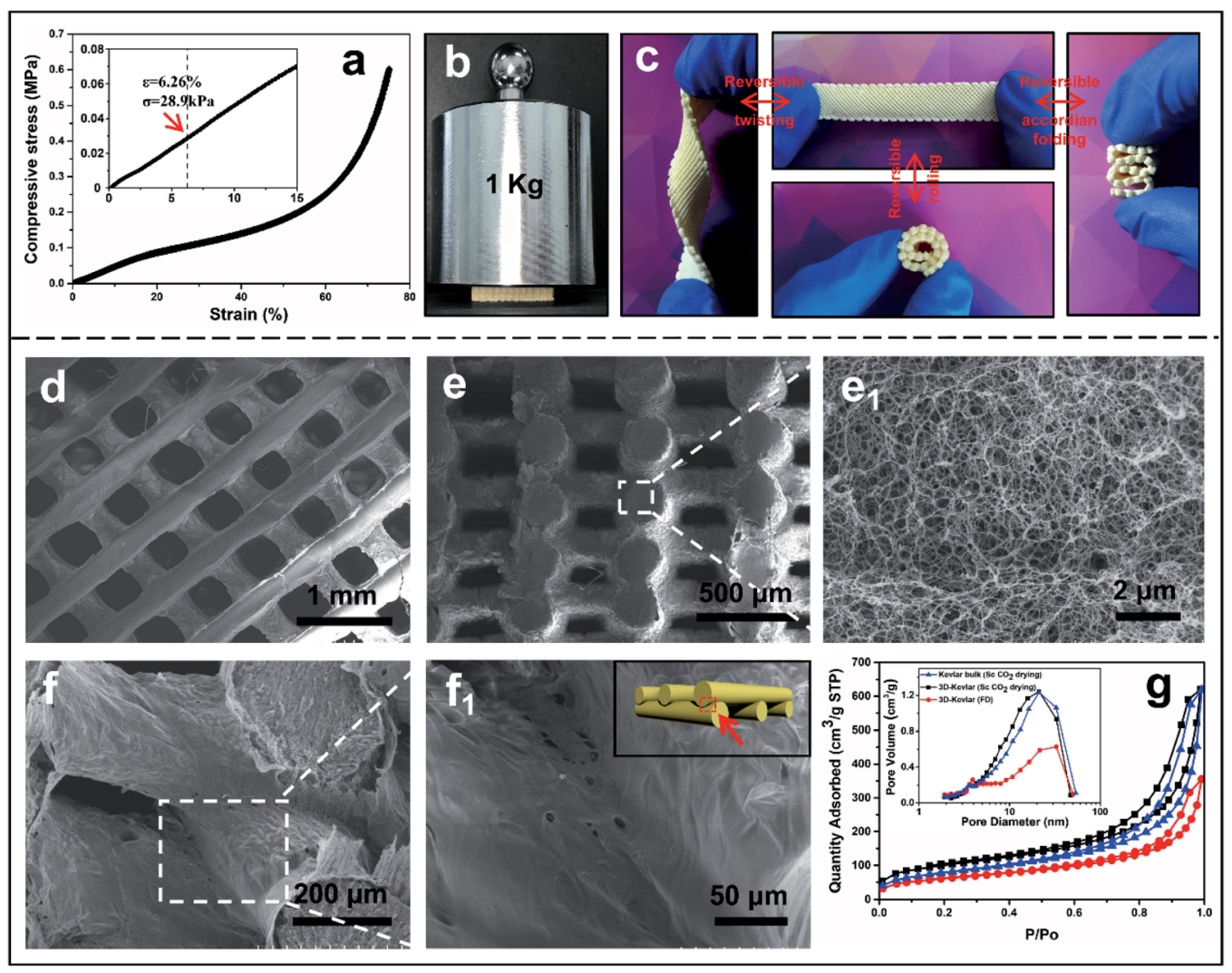

Fig. 2 (a) Compressive stress-strain curve measured from the 3D printed Kevlar aerogel woodpile (density of $26.8 \mathrm{mg} \mathrm{cm}^{-3}$ ). The inset is the corresponding enlarged portion of (a). (b) Digital photo of the Kevlar aerogel woodpile under a $9.8 \mathrm{~N}$ load, corresponding to the point $(\varepsilon=6.26 \%$, $\sigma=28.9 \mathrm{kPa}$ ) illustrated by the red arrow in (a). (c) Reversible twisting, subsequent folding and rolling of the Kevlar aerogel strip. (d-f) SEM images of the Kevlar aerogel woodpile from the top view (d), cross sectional view (e), enlarged cross section of a printed ANF strut ( $e_{1}$ ), the morphology between the adjacent struts $(f)$ and the enlarged junction $\left(f_{1}\right)$ illustrated by the red arrow. $(g)$ Nitrogen adsorption-desorption isotherms of the 3D printed Kevlar aerogel via different drying processes. The inset in $(\mathrm{g})$ is the corresponding pore volume. 
shown from the top view in Fig. $2 d$ and the cross section in Fig. 2e. The close-up micrograph of the cross section of a printed strut exhibited a fine and uniform interconnected porous network consisting of entangled nanofibres formed during the sol-gel process (Fig. 2 $\mathrm{e}_{1}$ ). Such a highly porous structure of the 3D woodpile manifests the unique capacity of DIW cryoprinting of DMSO-based ink, through which a well-defined hierarchically interconnected porous structure can be digitally printed, spanning from mesopores and small macropores within the struts to the large macropores between the adjacent struts. Another advantage is the strong adhesion between the printed layers generated by in situ welding during the cryoprinting process. As shown in Fig. 2f, the ANF struts were not loosely placed over each other but smoothly welded between the adjacent struts. It is envisaged that when freshly extruded ink was deposited on the top of the frozen layers, the warmer fluidic ink must have easily adhered to and slightly melted the frozen surface before being refrozen together at low temperature $(-10$ $\left.{ }^{\circ} \mathrm{C}\right) .{ }^{47}$ Such features of fused interconnection at the junctions between the neighboring ANF struts were thouroughly examined by SEM at different magnifications (Fig. $2 \mathrm{f}_{1}$ and $\mathrm{S} 17$ in ESI $\dagger$ ). The effect of the drying process on the specific surface area $\left(S_{\mathrm{BET}}\right)$ and pore volume distribution of the woodpile was tested by the BET method (Fig. 2g). The type IV nitrogen adsorption/desorption isotherm showed a hysteresis loop at relatively high pressure, indicating a large quantity of mesopores in the $3 \mathrm{D}$ network. The $S_{\mathrm{BET}}$ and pore volume were approximately $350 \mathrm{~m}^{2} \mathrm{~g}^{-1}$ and $0.959 \mathrm{~cm}^{3} \mathrm{~g}^{-1}$ for the samples via supercritical drying, higher than those via freeze-drying $\left(215 \mathrm{~m}^{2}\right.$ $\mathrm{g}^{-1}$ and $0.549 \mathrm{~cm}^{3} \mathrm{~g}^{-1}$ respectively, see Fig. $2 \mathrm{~g}$ ). The high efficiency of the supercritical drying process in maintaining the porous structure of the woodpile, even exceeded those of 3D printed graphene aerogels with a surface area of $130 \mathrm{~m}^{2} \mathrm{~g}^{-1}$ and nanofibrillated cellulose-nanopaper with a surface area of 304 $\mathrm{m}^{2} \mathrm{~g}^{-1}$ obtained by freeze- or supercritical drying. ${ }^{48,49}$ The inset in Fig. $2 \mathrm{~g}$ depicted the pore size distribution of the aerogel woodpile structure, which further confirmed the hierarchically porous feature. Besides, the X-ray diffraction (XRD) patterns and Fourier transform infrared (FTIR) spectra have shown negligible changes between Kevlar woodpile structures by different drying processes, indicating the stability of the chemical structure and crystallinity of the ANF aerogel (Fig. S18, ESI $\dagger$ ).

Poisson's ratio is one of the key elastic constants to describe the transverse deformation with respect to the longitudinal deformation of materials under normal stress conditions. Like most conventional materials, Kevlar fibres or bulk materials have a positive Poisson's ratio. ${ }^{50}$ On the other hand, auxetic materials with a negative Possion's ratio have been achieved through design and fabrication of specific internal structures, and the latest advancement of design and modelling also enabled the prediction and control of the polarity (negative or positive) and value of the Poisson's ratio of a newly designed material. ${ }^{18-20}$ To explore the feasibility of controlling Poisson's ratio and understand the response of Kevlar aerogel-based auxectic materials in a scalable manner, five types of porous architectures with predicted values of the Poisson's ratio from
-0.8 to 0.4 over large tensile deformations ${ }^{19,20,29}$ were designed and digitally fabricated by optimised $3 \mathrm{D}$ cryo-printing, and were validated against the numerically predicted behaviour (Fig. 3a and S19a in the ESI $\dagger$ ). Among them, a set of shape-optimised superellipses with theoretical Poisson's ratio values of -0.8 , -0.4 and 0 (Fig. S20, ESI $\dagger$ ), reported in the literature, ${ }^{20}$ have been adapted in this study and the architectures with positive $(0.4,0.1)$ and zero (0) Poisson's ratios were also studied for comparison.

The corresponding 3D Kevlar aerogel architectures with selected repeated unit cells were manufactured by cryo-printing and subsequent supercritical drying (Fig. $3 \mathrm{~b}$ and $\mathrm{S} 19 \mathrm{~b}$ in the ESI $\dagger$ ). The SEM images proved that the printed geometries matched the designed unit-cells with great accuracy (Fig. 3c and $\mathrm{S} 19 \mathrm{c}$ in the ESI $\dagger$ ). The snapshots of the mechanical response of these 3D printed ANF aerogel structures in Fig. 3d and S21 in the ESI and Movie S3, $\uparrow$ demonstrate that the different deformation behaviours occurred in axial and transverse directions simultaneously under the same tensile load conditions. Poisson's ratios as a function of the axial strain (up to 15\%) for each printed ANF aerogel structure were approximated by measuring the axial and transverse deformation, and summarized in Fig. 3e. It is clear that except for the case of theoretical $\nu=0.4$, non-positive Poisson's ratios $(\nu \leq 0)$ of the four types of the cellular structure are more or less constant, especially in the small strain region $(<10 \%)$, and also agree well with theoretical predictions by analytical models and deformation simulation..$^{19,20,29}$

In the cases of $\nu \leq 0$, such as the three typical topological microstructures in Fig. S20 in the ESI, $\uparrow$ significantly different Poisson's ratio behaviours were exhibited, demonstrating that the repeated unit-cell structures have more predominant effect on Poisson's ratios than the intrinsic elasticity of materials. As predicted, within the range of elastic deformation, Poisson's ratio is mainly contributed by the shape change of the internal architecture. Both axial and transverse elongations were engendered by remarkable angular changes (rotational deformation) of the interconnected struts around the central node during stretching while less notable change in length of the hinged struts, resulting in a more or less constant Poisson ratio. ${ }^{19}$ It is of note that a small increase in Poisson's ratio started in the case of $\nu=-0.8$ as the strain was further increased. In contrast, the experimental Poisson's ratio of the structure with a positive value of $\nu=0.4$ showed a straindependent profile, varying approximately from 0.99 to 2.08 in a logarithmic fashion (Fig. 3e). In this case, the significant lateral shrinkage observed in Fig. S21 in the ESI $\uparrow(\nu=0.4)$ was attributed to the actual lateral compressive stresses applied to the nodes along the struts (at $\pm 45^{\circ}$ ) of the square diamond shaped unit-cells under the uniaxial tensile conditions. Therefore, the digitally designed and printed cellular structure with different Poisson's ratio values clearly demonstrated the controlled deformation associated with the lateral expanding versus contracting behaviour of repeated unit-cell architectures, exhibiting the capacity of tuning the polarity and value of Poisson's ratio for 3D Kevlar aerogel architectures. To the best of our knowledge, this is the first time 3D auxetic architectures (i.e. metamaterials) have been fabricated using polymeric 


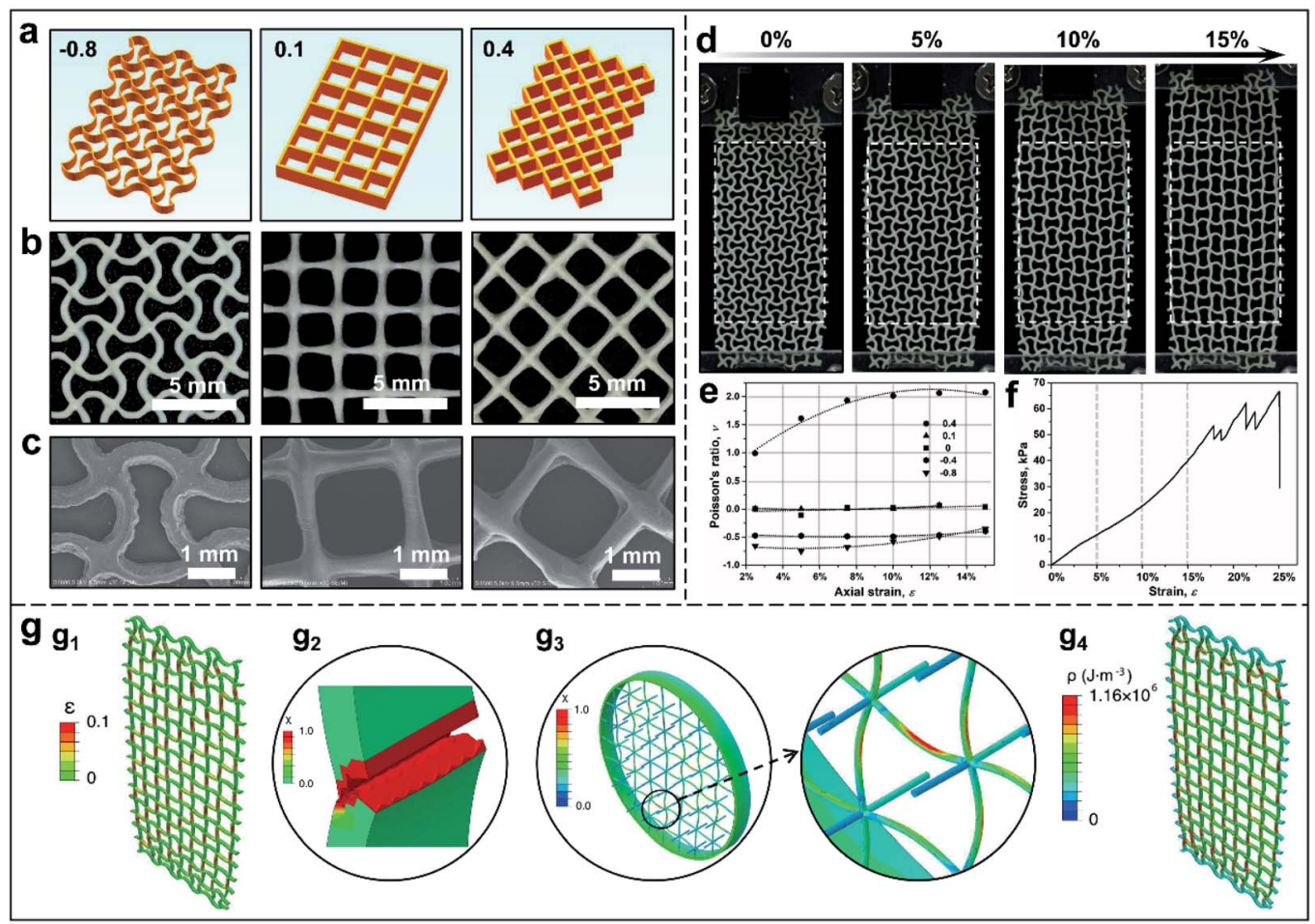

Fig. 3 Tensile mechanical properties of the 3D printed Kevlar aerogel auxetic architectures. (a-c) Designed models (a), printed unit-cells (b) and SEM images (c) of the corresponding architectures with theoretical Poisson's ratio $\nu=-0.8,0.1$ and 0.4. (d) Photos of deformation of the 3DKAAA $(\nu=-0.8)$ under a given longitudinal strain from $0 \%$ to $15 \%$. (e) Experimental Poisson's ratio of $3 D-K A A A s(\nu=-0.8,-0.4,0,0.1$ and 0.4$)$ as a function of the strain (points) and corresponding fitted curves (dashed line). (f) Tensile stress-strain curve of the 3D-KAAA ( $\nu=-0.8$ ). (g) Simulated strain distribution of the 3D-KAAA $(\nu=-0.8)$ at a $17.5 \%$ strain loading when the $1^{\text {st }}$ fracture occurs $\left(g_{1}\right)$, fracture parameter pattern of a damaged strut $\left(g_{2}\right)$, and a section of the single strut $\left(g_{3}\right)$ in the fracture process, and energy absorption distribution at a $16 \%$ strain before the $1^{\text {st }}$ fracture $\left(g_{4}\right)$

aerogels. Nevertheless, it is of note that there is discrepancy in the experimental value of Poisson's ratio compared with theoretical prediction, which could be attributed to (1) some minor pre-load and strain occurring in the samples before the tests; and (2) unavoidable geometrical and mechanical differences between the theoretical models and the printed samples.

The stress-strain profile in Fig. 3f reveals linear elasticity following multi-step fracture failure of the 3D-KAAA with $\nu=$ -0.8 and a density of $11.9 \mathrm{mg} \mathrm{cm}^{-3}$, reminiscent of the failure behaviour of continuous fibre reinforced composites. The ultimate tensile strength, $66 \mathrm{kPa}$, at a $25 \%$ fracture strain and elastic modulus, $238 \mathrm{kPa}$, were obtained respectively, four times stiffer than stretchable binary carbon aerogels (elastic modulus of $58 \mathrm{kPa}$ for $\left.9.7 \mathrm{mg} \mathrm{cm}{ }^{-3}\right) .{ }^{51}$ The superior tensile properties were achieved mainly by the synergistic effect of the hierarchically interconnected network of the aerogel architecture. A multi-scale extended finite element method (XFEM) was applied to model such highly porous structures to further reveal the mechanical mechanism (Fig. S22 and S23 in the ESI†). The simulation results show that most of the strain $(17.5 \%)$ at the first fracture was contributed by the deformation of the individual struts along the long axis of the load conditions rather than around the joint regions (Fig. $3 g_{1}$ ), implying the strong adhesion at the joints. The zig-zag fracture and multi-step failure mechanism also fit well with the simulated stress-strain curve (Fig. S24, ESI†). When a single strut in the auxetic architectures was broken up, the stress level dropped instantaneously, but could be sustained by the remaining struts, and they regained the strength under constant tensile load after each strut's break-up until the final catastrophic failure. Based on the extended model of regular interconnected beams for the printed struts in XFEM modelling, the simulated results showed that local stress concentration also led to a local zig-zag fracture surface (Fig. $3 \mathrm{~g}_{2}$ ) of individual strut at the microscale. In reality, the whole randomly entangled network of nanofibres could withstand more bending or buckling during the stretching, compared to the debonding and stress localization of straight beams simplified by modelling (Fig. $3 \mathrm{~g}_{3}$ ), which was widely observed in the debonding mechanism in other auxetic architectures. ${ }^{52-54}$ In the case of the 3DKAAA with $\nu=-0.8$, the strong adhesion between the struts and junctions, randomly-entangled nanofibre network within the struts and multi-stage fracture mechanism made such ultra-lightweight auxetic aerogel architectures tougher with more energy absorption (80.1 $\left.\mathrm{J} \mathrm{g}^{-1}\right)$ compared with the structure with a positive Poisson's ratio (e.g., $25.9 \mathrm{~J} \mathrm{~g}^{-1}$ for $\nu=0.4$ ) (Fig. $3 \mathrm{~g}_{4}$ and S25-S27 in the ESI $\dagger$ ).

By taking advantage of the unexpected control of the porosity and mechanical properties of the 3D-KAAA, various post-modifications have been conducted. Again, in the case of 
the 3D-KAAA with $\nu=-0.8$, a hydrophobic barrier layer of FC resin with a thickness of several nanometers was coated on the surface of the struts in order to improve its long-term stability. The FTIR spectra revealed that FC was coated on the surface of the 3D-KAAA successfully due to the appearance of the characteristic absorption peak of $-\mathrm{CF}_{3}$ at $1202 \mathrm{~cm}^{-1}$ (Fig. S28, ESI $\dagger$ ). The $S_{\mathrm{BET}}$ and pore volume of the 3D-KAAA after FC coating reduced to approximately $238 \mathrm{~m}^{2} \mathrm{~g}^{-1}$ and $0.676 \mathrm{~cm}^{3}$ $\mathrm{g}^{-1}$ from original $350 \mathrm{~m}^{2} \mathrm{~g}^{-1}$ and $0.959 \mathrm{~cm}^{3} \mathrm{~g}^{-1}$, respectively (Fig. S29, ESI $\dagger$ ), as a result of the smoother and flatter surface of the 3D-KAAA after treating with FC (Fig. 4a and S30 in the ESI $\dagger$ ). Despite the reduction of the surface and pore size, the FC coating caused little change in the mesoporous structure within the strut network or printed macropores as shown in Fig. S29b. $\dagger$ In contrast to the initial superhydrophilic properties of the uncoated 3D-KAAA (Fig. $4 \mathrm{a}_{1}$ ), the water droplet retained a nearly spherical shape on the surface of the FCcoated 3D-KAAA, with an approximate static contact angle of about $149^{\circ}$ (Fig. $4 \mathrm{a}_{2}$ ), which demonstrated an almost superhydrophobic performance. Interestingly, a water droplet (dyed with rhodamine B) could be transported from the upper surface to the lower surface through the expansion of the hydrophobic reentrant geometry during stretching-retracting (Fig. 4b and Movie $\mathrm{S} 4 \dagger$ ), which could be an inspiration for a new path to pump and transport aqueous droplets or moisture. Furthermore, the 3D-KAAA could be colored by soaking it directly in a solution of ethanol-soluble metal complex dye or fluorescent dye (rhodamine B). As a result, visible- (Fig. 4c) and ultraviolet-light (Fig. 4d) irradiated luminescent 3D-KAAAs were readily fabricated owing to their ultra high surface area. The colored 3D auxetic architectures exhibited little change in microstructure and specific surface area after re-drying (Fig. S31 and S32, ESI $\dagger$ ).

Finally a thermoresponsive phase change composite, 3DKAAA/PCM, was developed by impregnation of phase change materials (PCMs, PEG-2K or wax used in this study) into the 3D-KAAA. Thermogravimetric analysis (TGA) verified that the highly interconnected 3D auxetic architectures could hold high percentages of PCMs (93.5\% for PEG-2K and 91.8\% for wax) without any leakage due to the strong capillary force of the porous structures within the aerogel struts (Fig. S33, ESI $\dagger$ ). The SEM images further revealed that the PCMs had been trapped and infiltrated homogeneously within the mesopores and small macropores inside the struts without blocking the large macropores between the adjacent struts (Fig. S34, ESI $\dagger$ ). The DSC heating and cooling curves demonstrated the high energy storage properties with a melting phase change enthalpy of 135 $\mathrm{J} \mathrm{g}^{-1}$ and $186 \mathrm{~J} \mathrm{~g}^{-1}$, melting phase change onset at $40.9^{\circ} \mathrm{C}$ and $29.2^{\circ} \mathrm{C}$, and freezing phase change onset at $29.5^{\circ} \mathrm{C}$ and $52.2^{\circ} \mathrm{C}$ for $3 \mathrm{D}-\mathrm{KAAA} / \mathrm{PEG}-2 \mathrm{~K}$ and $3 \mathrm{D}-\mathrm{KAAA} /$ wax, respectively (Fig. 4e). Because of prompt response at the phase transition temperature of PCMs, 3D-KAAA/PEG-2K showed thermoresponsive shape memory behaviour of the $3 \mathrm{D}$ stretchable architectures driven by external thermal stimulation, as illustrated in Fig. $4 \mathrm{f}$

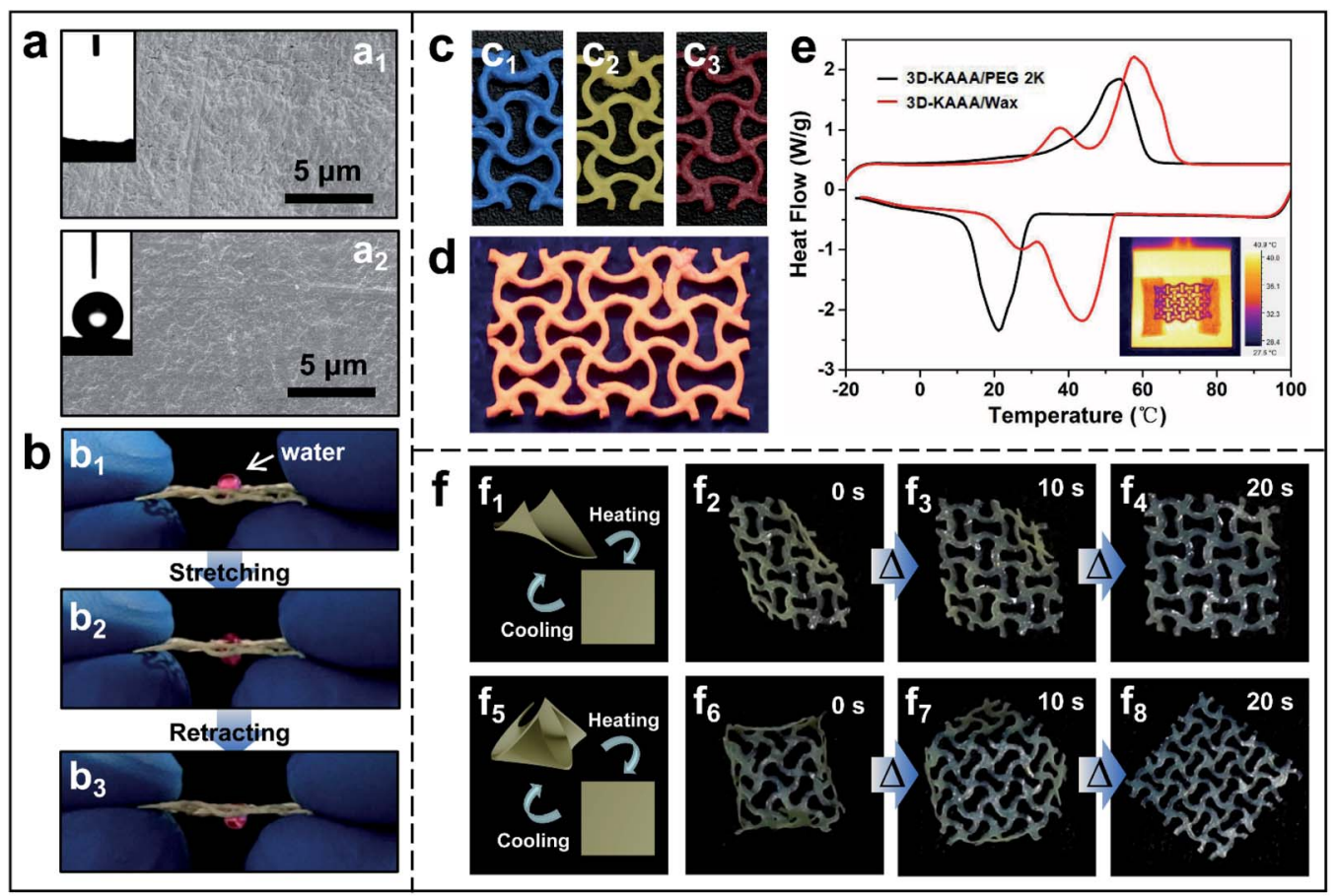

Fig. 4 The multi-functionalization of the 3D-KAAA. (a) SEM images of the 3D-KAAA before $\left(a_{1}\right)$ and after $\left(a_{2}\right)$ FC coating. The inset is the water contact angle of the corresponding surface. (b) Digital photos of a water droplet being transported from the upper surface $\left(b_{1}\right)$ to the lower surface $\left(b_{3}\right)$ during stretching-retracting. ( $c$ and $d$ ) Digital photos of the luminescent 3D-KAAA after soaking in the metal complex dye (c) or fluorescent dye (d) solution. (e) DSC curves of 3D-KAAA/PEG-2K and 3D-KAAA/wax composites. The inset in (e) is a thermal image of 3D-KAAA/ PEG-2K supported by two insulating foams. (f) Diagrams of deformation $\left(f_{1}\right.$ and $\left.f_{5}\right)$ and digital photos $\left(f_{2}-f_{4}\right.$ and $\left.f_{6}-f_{8}\right)$ of the $3 D-K A A A / P E G-2 K$ composite transformed from a temporarily bent shape $\left(f_{2}\right.$ and $\left.f_{6}\right)$ into a permanently flat shape $\left(f_{4}\right.$ and $\left.f_{8}\right)$ at $100{ }^{\circ} \mathrm{C}$. 
and Movie S5. $\uparrow$ The response time activated by the external heat was tracked by using a high-speed camera. The heated 3D auxetic architecture was initially deformed into a temporary shape of diagonal bending and frozen on a cold plate at about $0{ }^{\circ} \mathrm{C}$ subsequently (Fig. $4 \mathrm{f}_{2}$ ). As soon as the bent composite was heated at $100{ }^{\circ} \mathrm{C}$, it started expanding into a dome-shape within 10 seconds, triggered by the rapid phase transition of PEG-2K. The dome-shape is a characteristic synclastic behaviour of auxetic materials under bending. However, the bent composite then quickly relaxed to its original shape within $20 \mathrm{~s}$ (Fig. $4 \mathrm{f}_{4}$ ), due to the viscous fluidic state of PEG polymer chains above the phase transition temperature. ${ }^{55} \mathrm{~A}$ more complex temporary shape could be obtained, such as quadrilateral bending, which responded to the elevated temperature in a similar way under the same conditions (Fig. $4 \mathrm{f}_{5}-\mathrm{f}_{8}$ ). Such a rapid shape response to the heat stimulus in a time scale of a few seconds was repeated for several cycles with full recovery, exhibiting the superior thermal-mechanical stability. The robust and speedy thermoresponsive synclastic behaviour of 3D-KAAA/PCMs may offer a new range of sensors, actuators and devices.

\section{Conclusion}

In summary, 3D Kevlar aerogel auxetic architectures with superior mechanical properties, ultra-low densities and large surface areas have been successfully designed and fabricated by coupling direct ink writing and freeze-casting using DMSObased inks. This solvent ink-based additive manufacturing technique provides the advantages including higher freezing temperature with low energy consumption, a potentially vast variety of new ink formulations from both organic and inorganic materials and nanomaterials with better dispersibility in DMSO, and improved capacity of manufacturing complex structures and components with a higher resolution. Ultralightweight Kevlar aerogels are currently being exploited in many emerging fields. The 3D printed Kevlar aerogel auxetic architectures possess tunable in-plane negative Poisson's ratios by digital design and a broad hierarchy of porous structures consisting of repeated specific reentrant unit-cells. Facile multifunctionalization of the aerogel auxetic materials has been demonstrated by post coating and impregnation. The fluorocarbon resin coated hydrophobic auxetic architecture is capable of transporting water through the digitally printed channels during the stretch expansion. The visible- and ultraviolet-lightirradiated luminescent auxetic architectures are easily fabricated by the simple dip-coating process in a dye solution. Finally, thermoresponsive auxetic polymer composites have been developed by impregnation of phase change materials. The prompt thermal-induced auxetic shape memory and robust thermal-mechanical conversion promise potential application in artificial muscles, sensors, actuators, and soft robotics. The versatile $3 \mathrm{D}$ cryo-printing method in combination with a variety of organic solvent-based inks provides a new on-demand manufacturing platform for creating more multi-functional auxetic architectures and devices for a wider range of applications in the nearest future.

\section{Conflicts of interest}

There are no conflicts to declare.

\section{Acknowledgements}

This work was financially supported by the National Key Research and Development Program of China (2016YFA0203301), the Natural Science Foundation of Jiangsu Province (BK20170428 and BK20190232) and the Royal Society Newton Advanced Fellowship (NA170184). We are also thankful for the financial support by the UK Engineering and Physical Sciences Research Council (EPSRC EP/L020904/1, EP/M026884/ 1 and EP/R02961X/1).

\section{References}

1 M. A. Aegerter, N. Leventis and M. M. Koebel, Aerogels handbook, Springer Science \& Business Media, New York, USA, 2011.

2 J. Wang, D. Liu, Q. Li, C. Chen, Z. Chen, P. Song, J. Hao, Y. Li, S. Fakhrhoseini and M. Naebe, ACS Nano, 2019, 13, 78607870 .

3 B. Yao, S. Chandrasekaran, H. Zhang, A. Ma, J. Kang, L. Zhang, X. Lu, F. Qian, C. Zhu and E. B. Duoss, Adv. Mater., 2020, 1906652.

4 B. Cai and A. Eychmüller, Adv. Mater., 2019, 31, 1804881.

5 P. Hu, J. Lyu, C. Fu, W. Gong, J. Liao, W. Lu, Y. Chen and X. Zhang, ACS Nano, 2019, 14, 688-697.

6 R. Wordsworth, L. Kerber and C. Cockell, Nature Astronomy, 2019, 3, 898-903.

7 A. J. Westphal, R. M. Stroud, H. A. Bechtel, F. E. Brenker, A. L. Butterworth, G. J. Flynn, D. R. Frank, Z. Gainsforth, J. K. Hillier and F. Postberg, Science, 2014, 345, 786-791.

8 D. Li, J. Ma, L. Dong and R. S. Lakes, Phys. Status Solidi, 2017, 254, 1600785.

9 K. Yoonho, Y. Hyunwoo, R. Zhao, S. A. Chester and X. Zhao, Nature, 2018, 558, 274-279.

10 S. Babaee, J. Shim, J. C. Weaver, E. R. Chen and K. Bertoldi, Adv. Mater., 2013, 25, 5044-5049.

11 Y. J. Ma, X. F. Yao, Q. S. Zheng, Y. J. Yin, D. J. Jiang, G. H. Xu, F. Wei and Q. Zhang, Appl. Phys. Lett., 2010, 97, 061909.

12 R. Lakes, Science, 1987, 235, 1038-1040.

13 C. Weng, Z. Dai, G. Wang, L. Liu and Z. Zhang, ACS Appl. Mater. Interfaces, 2019, 11, 6541-6549.

14 K. L. Alderson and K. E. Evans, Polymer, 1992, 33, 4435-4438. 15 R. Lakes, Adv. Mater., 1993, 5, 293-296.

16 Y. Wen, E. Gao, Z. Hu, T. Xu, H. Lu, Z. Xu and C. Li, Nat. Commun., 2019, 10, 2446.

17 X. Jiang, S. Luo, L. Kang, P. Gong, W. Yao, H. Huang, W. Li, R. Huang, W. Wang and Y. Li, Adv. Mater., 2015, 27, 48514857.

18 T. Bückmann, N. Stenger, M. Kadic, J. Kaschke, A. Frölich, T. Kennerknecht, C. Eberl, M. Thiel and M. Wegener, Adv. Mater., 2012, 24, 2710-2714.

19 D. Y. Fozdar, P. Soman, J. W. Lee, L. Han and S. Chen, Adv. Funct. Mater., 2011, 21, 2712-2720. 
20 A. Clausen, F. Wang, J. S. Jensen, O. Sigmund and J. A. Lewis, Adv. Mater., 2015, 27, 5523-5527.

21 K. Bertoldi, P. M. Reis, S. Willshaw and T. Mullin, Adv. Mater., 2010, 22, 361-366.

22 M. Taylor, L. Francesconi, M. Gerendás, A. Shanian, C. Carson and K. Bertoldi, Adv. Mater., 2014, 26, 2365-2370.

23 B. Xu, F. Arias, S. T. Brittain, X. M. Zhao, B. Grzybowski, S. Torquato and G. M. Whitesides, Adv. Mater., 1999, 11, 1186-1189.

24 J. Valente, E. Plum, I. J. Youngs and N. I. Zheludev, Adv. Mater., 2016, 28, 5176-5180.

25 A. Ulvestad, A. Singer, J. Clark, H. Cho, J. Kim, R. Harder, J. Maser, Y. Meng and O. Shpyrko, Science, 2015, 348, 1344-1347.

26 Y. Ma, Y. Zheng, H. Meng, W. Song, X. Yao and H. Lv, J. Mech. Behav. Biomed. Mater., 2013, 23, 22-31.

27 Q. Zhang, X. Xu, D. Lin, W. Chen, G. Xiong, Y. Yu, T. S. Fisher and H. Li, Adv. Mater., 2016, 28, 2229-2237.

28 X. Xu, Q. Zhang, M. Hao, Y. Hu, Z. Lin, L. Peng, T. Wang, X. Ren, C. Wang and Z. Zhao, Science, 2019, 363, 723-727.

29 P. Yan, E. Brown, Q. Su, J. Li, J. Wang, C. Xu, C. Zhou and D. Lin, Small, 2017, 13, 1701756.

30 C. Zhu, T. Y.-J. Han, E. B. Duoss, A. M. Golobic, J. D. Kuntz, C. M. Spadaccini and M. A. Worsley, Nat. Commun., 2015, 6, 6962.

31 C. Zhu, T. Liu, F. Qian, T. Y. Han, E. B. Duoss, J. D. Kuntz, C. M. Spadaccini, M. A. Worsley and Y. Li, Nano Lett., 2016, 16, 3448-3456.

32 G. Li, G. Hong, D. Dong, W. Song and X. Zhang, Adv. Mater., 2018, 30, 1801754.

33 W. He, G. Li, S. Zhang, Y. Wei, J. Wang, Q. Li and X. Zhang, ACS Nano, 2015, 9, 4244-4251.

34 J. Lyu, Z. Liu, X. Wu, G. Li, D. Fang and X. Zhang, ACS Nano, 2019, 13, 2236-2245.

35 Z. Liu, J. Lyu, D. Fang and X. Zhang, ACS Nano, 2019, 13, 5703-5711.

36 M. Yang, K. Cao, L. Sui, Y. Qi, J. Zhu, A. Waas, E. M. Arruda, J. Kieffer, M. D. Thouless and N. A. Kotov, ACS Nano, 2011, 5, 6945-6954.

37 V. T. Naidu, International Research Journal of Engineering and Technology, 2017, 4, 3466-3473.
38 J. Zhu, W. Cao, M. Yue, Y. Hou, J. Han and M. Yang, ACS Nano, 2015, 9, 2489-2501.

39 H. Huang, G. Zhao, Y. Zhang, J. Xu, T. L. Toth and X. He, ACS Biomater. Sci. Eng., 2017, 3, 1758-1768.

40 V. Li, A. Mulyadi, C. Dunn, Y. Deng and H. J. Qi, ACS Sustain. Chem. Eng., 2017, 6, 2011-2022.

41 Y. Jiang, Z. Xu, T. Huang, Y. Liu, F. Guo, J. Xi, W. Gao and C. Gao, Adv. Funct. Mater., 2018, 28, 1707024.

42 Y. Si, J. Yu, X. Tang, J. Ge and B. Ding, Nat. Commun., 2014, 5, 5802.

43 H. Maleki, S. Montes, N. Hayati-Roodbari, F. Putz and N. Huesing, ACS Appl. Mater. Interfaces, 2018, 10, 2271822730.

44 S. Barg, F. M. Perez, N. Ni, P. do Vale Pereira, R. C. Maher, E. Garcia-Tuñon, S. Eslava, S. Agnoli, C. Mattevi and E. Saiz, Nat. Commun., 2014, 5, 4328.

45 X. Zhang, Z. Sui, B. Xu, S. Yue and B. Liu, J. Mater. Chem., 2011, 21, 6494-6497.

46 G. Li, M. Zhu, W. Gong, R. Du, A. Eychmüller, T. Li, W. Lv and X. Zhang, Adv. Funct. Mater., 2019, 29, 1900188.

47 Q. Zhang, F. Zhang, S. P. Medarametla, H. Li, C. Zhou and D. Lin, Small, 2016, 12, 1702-1708.

48 R. M. Hensleigh, H. Cui, J. S. Oakdale, C. Y. Jianchao, P. G. Campbell, E. B. Duoss, C. M. Spadaccini, X. Zheng and M. A. Worsley, Mater. Horiz., 2018, 5, 1035-1041.

49 H. Sehaqui, Q. Zhou, O. Ikkala and L. A. Berglund, Biomacromolecules, 2011, 12, 3638-3644.

50 F. Xu, W. Fan, Y. Zhang, Y. Gao, Z. Jia, Y. Qiu and D. Hui, Composites, Part B, 2017, 116, 398-405.

51 F. Guo, Y. Jiang, Z. Xu, Y. Xiao, B. Fang, Y. Liu, W. Gao, P. Zhao, H. Wang and C. Gao, Nat. Commun., 2018, 9, 881.

52 M. Peng, Z. Wen, L. Xie, J. Cheng, Z. Jia, D. Shi, H. Zeng, B. Zhao, Z. Liang and T. Li, Adv. Mater., 2019, 31, 1902930.

53 E. L. M. Ribolla, M. R. Hajidehi, P. Rizzo, G. F. Scimemi, A. Spada and G. Giambanco, Structure \& Infrastructure Engineering, 2017, 14, 801-816.

54 C. Gao, V. Slesarenko, M. C. Boyce, S. Rudykh and Y. Li, Sci. Rep., 2018, 8, 11834.

55 F. Guo, X. Zheng, C. Liang, Y. Jiang, Z. Xu, Z. Jiao, Y. Liu, H. Wang, H. Sun and L. Ma, ACS Nano, 2019, 13, 5549-5558. 